\title{
Treatment with Doxycycline is Associated with Reduced Spleen Size in Hyper-Reactive Malarial Splenomegaly: A Cohort Study
}

Peter John Barnard Davies ( $\sim$ peter.davies5@nhs.net )

Kagando Hospital https://orcid.org/0000-0003-0208-313X

Simon Eyre

Kagando Hospital

\section{Research}

Keywords: Malaria, hyper-reactive splenomegaly, HMS, doxycycline, Uganda

Posted Date: May 8th, 2020

DOI: https://doi.org/10.21203/rs.3.rs-26506/v1

License: (c) (i) This work is licensed under a Creative Commons Attribution 4.0 International License.

Read Full License 


\section{Abstract}

\section{Background:}

Hyper-reactive malarial splenomegaly (HMS) is a poorly understood sequela of chronic parasitaemia, caused by an aberrant immune response, and confers a high associated mortality. There arefew historical data on effective treatments, which are either unavailable or unaffordable in endemic areas. In Kagando, Western Uganda patients were treated with Doxycycline and followed up to measure the effect.

\section{Methods:}

Patients were identified by medical officers from March 2018 at Kagando Hospital who met Fakunle's criteria for HMS; children under 12 years and pregnant women were excluded. The baseline full blood count and splenic size from the costal margin was measured and manually recorded. Patients were discharged with a 6-monthcourse of Doxycycline and asked to attend on completion for follow-up assessment. Spleenmeasurements and compliance wererecorded by medical officers, before being tabulated and analysed electronically.

\section{Results:}

In total 27 patients were treated for HMS over a two year period with 7 patients returning for a follow up between 6-15 months. The median age was 36 years with a near equal male to female ratio. Prior to treatment patients displayed a variable degree of pancytopenia with a median haemoglobin of 10.1, leucocytes 4.5 and platelets 82 , and the median spleen size was $16 \mathrm{~cm}$ from the costal margin. The Wilcoxon signed-rank test was used for statistical analysis. There was a significant reduction in spleen size $p<0.05$, with $4 / 7$ showing complete resolution. There was a numericalimprovement in platelet count (median82 vs 125) $p=0.16$.

\section{Conclusions:}

Regular doxycycline use as malaria prophylaxis in a holoendemic area of Uganda was associated with a significant reduction in spleen size and a numerical improvement in platelet count. A larger cohort study and an improved follow-up regime is required to further characterise this effect. However, doxycyclineprovidesa practical and affordable treatment modality in a rural Ugandan population, with no current national or international guidelines. In some patients the benefit is seen up to a year from treatment completion.

\section{Background}

Hyper-reactive malarial splenomegaly syndrome (HMS) is the major cause of massive splenomegaly or tropical splenomegaly syndrome in malaria-endemic countries. The distribution reflects global malaria distribution with no data of prevalence in Uganda, East Africa or indeed worldwide. Estimates of population prevalence are historical and, indeed, highly variable ranging from 800 cases per 1000 in 
areas of Papua New Guinea to 1.6 per 1000 in the Gambia (,). Massive splenomegaly is a common finding at Kagando Mission Hospital, a rural hospital in western Uganda.

The syndrome is thought to reflect an aberrant immune response to persistent malarial parasitaemia. The pathophysiology is based in a hypergammaglobulinemia in response to chronic or repeated malaria exposure. The result is B-lymphocyte over-production of $\operatorname{lgM}$, and cryoglobulin formation that causes chronic activation of the reticular-endothelial system resulting in progressive splenomegaly (,).

Traditionally the Hackett criteria (I-V) was used to stratify spleen size on palpation in relation to the costal margin and umbilicus (). Whereas, the Fakunle criteria specifies a spleen size of $>10 \mathrm{~cm}$ below the costal margin plus other clinical criteria including elevated IgM titre, a presumptive diagnosis and a favourable response to prophylaxis (). Modern stratification utilises an ultrasound criteria of spleen size $>16 \mathrm{~cm} \mathrm{().}$

Malaria is sub-acute and patients commonly exhibit pancytopenia in keeping with hypersplenism. The immune impairment and subsequent sepsis, in addition to splenic rupture, is the basis of the high associated mortality (4). This was reported as $37 \%$ at 6 years in Papua New Guinea, increasing with spleen size in a case series of 75 adults, and $26 \%$ at 10 years in another study (,). Despite this there is remarkably little evidence to support specific treatment regimes. In imported cases a short term treatment in the absence of re-infection may be sufficient for resolution. Yet there is no consensus for treatment duration or indeed drug choice in endemic countries (). The most established evidenced treatment is with either weekly Chloroquine or daily Proguanil (4,). However, chloroquine is unavailable due to widespread resistance and Proguanil is poorly available and prohibitively expensive. Doxycycline has not previously been used in an endemic country, despite availability, good safety profile and efficacy as prophylaxis (,).

At Kagando Mission Hospital, Western Uganda, a 6-month course of Doxycycline has been adopted as the prophylactic treatment in those with presumptive HMS. This retrospective evaluation aimed to assess the efficacy of this regime in HMS by contrasting biochemical and clinical parameters at baseline and following 6-month treatment.

\section{Methods}

Kagando mission hospital lies in Kisinga county at the foot of the Rwenzori Mountains in Western Uganda. In total $75.5 \%$ of the population live rurally in small village communities on subsistence agriculture. There is a catchment population of 40,631 in the sub-county, expanding at a rate of $2.45 \%$ per year () . There is a continuous malaria transmission with peak incidence during biannual rainy seasons March-April and October-November. Local experience suggests a low local prevalence of schistosomiasis and visceral leishmaniasis. Whereas, malaria was the primary diagnosis in $23 \%$ of all adult hospital admissions between February - May 2018.

A treatment regime was instigated for those presenting at Kagando Hospital with HMS in early 2018. Approval for patient analysis was given by the hospital management committee. The hospital organised appropriate medical education for doctors and medical officers prior to instigating this protocol. 
Patients presenting to Kagando with gross splenomegaly, fitting Fakunle's criteria (spleen size $>10 \mathrm{~cm}$ from costal margin and presumptive diagnosis of HMS) were discharged with a 6-month course of once daily doxycycline $100 \mathrm{mg}$. The spleen size was measured by Medical Officers (MO) in the outpatient department (OPD) and a full blood count (FBC) were measured at baseline. Patient's basic demographic details were recorded and a follow up date was provided for 6 months. Blood and clinical parameters were measured at follow up, in addition to a verbal assessment of compliance. All of these aspects of care including documentation were performed by the MOs in the OPD. Physicians would liaise with the relevant MOs as required if inpatients were referred for treatment and follow up.

Exclusion criteria include children under the age of 12 years old and pregnancy where doxycycline is contraindicated. Women of childbearing age were counselled to avoid pregnancy, use contraception or stop the treatment if there was any uncertainty. Other possible causes of massive splenomegaly were excluded including assessment for sickle cell disease in younger patients, lymphoproliferative disorders and schistosomiasis if patients had been exposed to locally infested lake water.

The data was tabulated and then reviewed retrospectively after a two year period was transferred to an anonymised electronic document for analysis. Baseline data was descriptive in nature. The Wilcoxon signed-rank test was used for statistical analysis of blood parameters and spleen size.

\section{Results}

Across two years, 29 patients with a clinical diagnosis of HMS received a 6 month course of doxycycline. Of this group three patients were excluded from the analysis, two had insufficient spleen size $(<10 \mathrm{~cm})$ as per the hospital guidance and one patient had no documented spleen measurement. Patient data was compiled as a handwritten document by the lead Medical Officer in OPD, providing consistency. Intermittently FBCs were unavailable due to faulty laboratory equipment. This resulted in five patients pretreatment and one patient post-treatment not having documented blood test results. In total seven patients returned for follow up. One of these was reviewed by an untrained medical officer, who documented spleen size as "slightly palpable", foregoing accurate comparison. This data is comprehensively laid out in Table 1. 
Table 1

Post-treatment blood tests, spleen size with interval change and the follow up duration are displayed below. The median measurements pre-treatment are included to allow direct comparison. $\mathrm{Hb}-$ ref. range

male 13.5-18 female 115-16.5, MCV ref. 80-100, WCC - 4-11, PLTS - platelets 150-450. Rx treatment.

\begin{tabular}{|llllllllll|}
\hline $\mathbf{n}$ & Age & Sex & $\begin{array}{l}\text { Hb } \\
\mathbf{g} / \mathbf{L}\end{array}$ & $\begin{array}{l}\text { MCV } \\
\mathbf{f L}\end{array}$ & $\begin{array}{l}\text { WCC } \\
\mathbf{x} 10^{9}\end{array}$ & $\begin{array}{l}\text { Plts } \\
\mathbf{x} 10^{9}\end{array}$ & $\begin{array}{l}\text { Spleen } \\
\text { size } \\
\mathbf{( c m})\end{array}$ & $\begin{array}{l}\text { Spleen } \\
\text { change } \\
(\mathbf{c m})\end{array}$ & $\begin{array}{l}\text { Follow up } \\
\text { interval } \\
\text { (days) }\end{array}$ \\
\hline 1 & 50 & F & NA & NA & NA & NA & 11 & -5 & 188 \\
\hline 2 & 35 & M & 12.2 & 91 & 1.32 & 156 & 0 & -14 & 216 \\
\hline 3 & 31 & F & 13.9 & 105.1 & 3.1 & 102 & 0 & -12.5 & 177 \\
\hline 4 & 36 & F & 11.5 & 96 & 3.27 & 70 & $\begin{array}{l}\text { NA (pre } \\
=14 \text { cm) }\end{array}$ & 454 & \\
\hline 5 & 47 & M & 16.9 & 89 & 3.96 & 127 & 0 & -16 & 435 \\
\hline 6 & 37 & M & 15.6 & 96 & 3.9 & 120 & 0 & -16 & 349 \\
\hline 7 & 18 & M & 14.6 & 86 & 5.7 & 105 & 4 & -14 & 210 \\
\hline $\begin{array}{l}\text { Median } \\
\text { pre-Rx }\end{array}$ & 36 & & 12.4 & 90.4 & 5.3 & 61 & 16 & NA & NA \\
\hline $\begin{array}{l}\text { Median } \\
\text { post-Rx }\end{array}$ & NA & & 14.3 & 93.5 & 3.6 & 125 & 0 & -14 & 216 \\
\hline
\end{tabular}

The median age was 36 years old with a slight female preponderance. The FBC demonstrated a mild microcytic pancytopenia with median haemoglobin of 10.1 ( $\mathrm{Hb}$ ref. male 13-18 g/L female 11.5-16.5), mean cell volume 83 (MCV ref. 80-100 fL), leucocytes 4.5 (WCC ref. $4-11 \times 10^{9} / \mathrm{L}$ ) and platelets of 82 (ref. $140-450 \times 10^{9}$ ). The median spleen measurement was $16 \mathrm{~cm}$. The patients who attended follow up are detailed in Table 1. There was a numerical improvement in haemoglobin levels and a marginal rise in MCV. Although there was a marked improvement in platelet count between groups, this was not statistically significant, $p=0.16$. Spleen size was markedly reduced in all patients with 4 of 7 impalpable at follow up and the median measurement at the costal angle reducing from $16 \mathrm{~cm}$ to $0 \mathrm{~cm}$. Six patients with valid splenic measurements were contrasted pre and post-treatment, confirming a significant reduction in spleen size $(p<0.05)$.

Although patients were instructed to return for 6 months follow up it is clear that this was impractical with the low number of returnees. Although 3 patients presents approximately six months from their treatment initiation, the range was up to 14.9 months and the median was 7.1 months. This is likely due to opportunistic visits from a poor and relatively immobile population. Finally, at follow up duration of treatment was confirmed and all 7 patients had received 6 months of doxycycline.

\section{Discussion}


Great strides have been made in tackling malaria as a result of the millennium development goal $6 \mathrm{c}$ that aimed to halt or reverse the incidence of malaria by 2015 . Consequently, malaria incidence has fallen by $37 \%$ worldwide and death rates by $60 \%$. The Global technical strategy for malaria aims for a further $90 \%$ reduction in the malaria incidence by 2030 (). Ultimately this strategy will underpin the decline in prevalence of HMS through prevention. However, until malaria incidence is sufficiently ameliorated a reasonable and affordable treatment strategy is required for HMS, given the significant mortality. Both established evidence based treatments Chloroquine and Proguanil are not available in the area, and the latter is too expensive for long term treatment in these patients comprised mainly of subsistence farmers. It is clear that a pragmatic and effective treatment is required.

Doxycycline is highly effective in providing malaria prophylaxis with efficacy between $84-96 \%$ in p.falciparum, higher in p.vivax (). It is highly effective as a blood schizontacidal agent, with partial activity as a causal prophylactic (against the liver stage). It is generally well tolerated with few side effects, few drug interactions and can be taken long term. A treatment for 6 months is not uncommon in dermatological conditions such as acne vulgaris and lymphatic filariasis (). The hospital had selected a course of 6 months in the absence of national or international guidelines on the subject, and the understanding that patients would likely be quickly re-exposed following treatment. It was felt that 6 months would give sufficient time for immune and splenic recovery, although alternative courses could be considered in the absence of reliable data.

Doxycycline use previously in HMS is limited and in non-endemic populations. One European case report used Malarone for malaria treatment followed by doxycycline for 30 days, resulting in resolution of splenomegaly (). However recent developments suggest prophylaxis in a non-endemic area is unnecessary if standard curative treatment is administered in the absence of re-exposure (16). In a retrospective case series of HMS ex-patriots and immigrants in Italy, patients were treated with a standard short course for acute malaria, and doxycycline was prescribed only in those visiting their countries of origin for the duration of their stay. Of those who took regular prophylaxis, $8 / 9$ were "improved or cured", whereas $10 / 13$ who took "intermittent treatment instead" were similarly "improved or cured"; presumably this was in terms of spleen size, blood results are not specified (16). Although this is a different population, returning to different risk areas, this suggests some efficacy. The group taking intermittent treatment are hard to characterise as this would depend on malaria endemicity, and the frequency and nature of their treatment. It is clearly practically and financially impossible to advocate lifelong prophylaxis in Kagando. A 6-month course provides a reasonable clearance time to allow recovery and could be re-instated, as a pulsed treatment, depending on refractory splenomegaly or recurrence following withdrawal. It is interesting that improvement persisted in our cohort even in the patients who represented late.

Consideration needs to be given to the antibiotic and anti-inflammatory effect of doxycycline. It has broad spectrum bacteriostatic activity and with efficacy against numerous tropical infections including rickettsia and leptospirosis. In theory this could afford these patients additional protection given that overwhelming sepsis is the major component of the significant associated mortality in HMS. Clearly this 
is unique to antibiotic prophylaxis. Furthermore, doxycycline has an anti-inflammatory effect, even in subantimicrobial levels. A decrease in inflammatory mediated cytokines as a result could create synergy in mediating the aberrant immune response in HMS (,). This mirrors the immune-regulatory effect of chloroquine in HMS where efficacy has been noted even in resistant $p$. falciparum infections ().

The logistics of follow up in rural western Uganda are a significant issue. The population primarily consists of farmers working in subsistence agriculture, who would struggle to afford travel to hospital and tests unless acutely unwell. This is the likely cause for those lost to follow up, coupled with a lack of infrastructure to facilitate this from the institution. A minority returned at their agreed follow up date, others returned opportunistically at a later date. This results in variability, in terms of time off prophylaxis and re-exposure to malaria, in a presumed high-risk environment. Despite this the spleen size and blood parameters demonstrated marked improvement even after a significant number of months from when the prophylaxis finished.

Another point of consideration is lack of diagnosis for the initial presentation to hospital. Patients are likely to have received a 3 day course of ACT for acute malaria in view of their clinical condition, positive rapid diagnostic test or blood film, yet this is not recorded. Whilst, a co-infection or another febrile tropical illness could have resulted in the presentation and affected the blood results. For instance Kagando is unable to locally test for arboviruses. Furthermore, although the majority of patients were treated by an experienced Medical Officer, due to staff turnover, later patients were not. There will be an element of subjectivity to spleen measurement and a margin of error, possibly more so in later returns. However the majority of returnees were found to have an impalpable spleen.

Although patients continue to be treated in Kagando in this manner, without funding and close oversight patient follow up will remain sporadic. Ideally patients would be recruited in an outpatient setting with no acute illness, yet patient identification would be limiting. Therefore, an attractive strategy would be to call the patients near their agreed 6-month appointment and subsidise travel and investigations. Alternatively, assessment could take place at local clinics and the results phoned through to Kagando, yet this would still require funding for phone credit charges and investigation costs. Certainly, formalisation and facilitation of follow up with a resultant expansion of returns would allow more accurate analysis and firmer conclusions. It would be desirable to know if these benefits were then lasting, how long for and the impact on mortality.

\section{Conclusions}

This intervention at Kagando was born out of necessity in identifying an affordable treatment strategy in an under-researched area of tropical medicine for a poor population, which, if untreated conveys a high mortality. Although the sample population was small there was a significant decrease in the spleen size and an improved trend in the pancytopenia in the blood results. These benefits were, in some patients, seen after 6 months from treatment completion. Further work is required to explore the effect of prophylactic doxycycline monotherapy on HMS, yet these results suggest efficacy in this strategy. 


\section{Abbreviations}

HMS: hyperreactive malaria splenomegaly, $\mathrm{Hb}$-haemoglobin, MCV-mean cell volume, WCC-white cell count, PLTs-platelet count, ACT-artesunate combination therapy, MO-medical officer, OPD-outpatient department, FBC-full blood count, Rx - treatment.

\section{Declarations}

\section{Consent for publication -}

Not applicable.

\section{Ethics approval and consent -}

Ethics approval was not required as this was a retrospective service evaluation of an established practice. Permission for data analysis was obtained from the hospital management committee.

\section{Availability of data and materials -}

All data generated or analysed during this study are included in this published article and its supplementary information.

\section{Competing interests -}

None to declare.

\section{Funding -}

None to declare.

\section{Author's contributions -}

PD analysed the data and was the main contributor to the paper. SE provided supervising guidance, analysis and editing.

\section{Acknowledgements -}

The authors would like to acknowledge Bezaleri working as a medical officer at Kagando. Dr Rob Morris the medical deputy director of Kagando and Dr Oliver Penney who helped facilitate data collection. In 
addition to the many hard working nurses, doctors and medical officers who provide excellent care for patients at Kagando, with limited resources.

\section{References}

1. Bhatia K, Crane G. HLA and tropical splenomegaly syndrome in the Upper Watut Valley of Papua New Guinea. Hum Immunol. 1985;13(4):235-242.

2. Greenwood BM, Groenendaal F, Bradley AK, Greenwood AM, Shenton F, Tulloch S et al. Ethnic differences in the prevalence of splenomegaly and malaria in The Gambia.Ann Trop Med Parasitol. 1987;81(4):345-54.

3. Hoffman SL, Carlos C, CampbellN, White J. Malaria. In: Guerrant R, Walker D, Weller P, editors.Drs. Richard L. Guerrant, David H. Walker, and Peter F. Weller Drs. Richard L. Guerrant, David H. Walker, and Peter F. Weller Tropical Infectious Diseases (Third Edition), W. B Saunders; 2011. P. 646-675.

4. Leoni S,Buonfrate D, Angheben A, Gobbi F, Bisoffi Z. The hyper-reactive malarial splenomegaly: a systematic review of the literature. Malar J. 2015;14:185.

5. Hackett LW. Spleen Measurement in Malaria. Natl Malar Soc. 1944; 3 (2): p.121-33.

6. Fakunle YM. Tropical splenomegaly. Part 1: Tropical Africa. Clin Haematol. 1981;10:963-75.

7. Crane GG, Wells JV, Hudson P. Tropical splenomegaly syndrome in New Guinea. I. Natural history. Trans R Soc Trop Med Hyg. 1972;66:724-32.

8. Crane G. Recent studies of hyperreactive malarious splenomegaly (tropical splenomegaly syndrome) in Papua New Guinea. P N G Med J. 1986;29:35-40.

9. Eseme F, Lodesani C, Amicarelli E, Marocco S, Monteiro G, Anselmi M, et al. Hyperreactive malarial splenomegaly in Europeans. Giornale Italiano di Medicina Tropicale. 2004;9:61-4.

10. McGregor, A, Doherty T, Lowe P, Chiodini P, Newsholme W. Hyperreactive Malarial Splenomegaly Syndrome-Can the Diagnostic Criteria Be Improved? Am J trop med hyg. 2015; 93 (3): 573-576.

11. Camara B, Kantambadouno JB, Martin-Blondel G, Berry A, Alvarez M, Benoit-Vical F, et al. Hyperreactive malarial splenomegaly: Three clinical cases and literature review. Med Mal Infect. 2009;39:29-35.

12. Bisoffi Z, Leoni S, Angheben A, Beltrame A, Eseme FE, Gobbi F, et al. Chronic malaria and hyperreactive malarial splenomegaly: a retrospective study on the largest series observed in a nonendemic country. Malar J. 2016; 15: 230.

13. Kasese district council: District profile and census data. 2014. http://kasese.go.ug/generalinformation/community-based-development/ Accessed $23^{\text {rd }}$ April 2020.

14. World Health Organisation: Achieving the malaria MDG target: reversing the incidence of malaria 2000-2015. 2015. https://www.who.int/malaria/publications/atoz/9789241509442/en/ Accessed $23^{\text {rd }}$ April 2020.

15. Tan KR, Magill AJ, Parise M, Arguin P. Doxycycline for Malaria Chemoprophylaxis and Treatment: Report from the CDC Expert Meeting on Malaria Chemoprophylaxis. Am J Trop Med Hyg. 2011 Apr 5; 
84(4): 517-531.

16. The Republic of Uganda: National Clinical Guidelines. 2016.

https://www.health.go.ug/sites/default/files/Uganda\%20Clinical\%20Guidelines\%202016_FINAL.pdf Accessed 24 $4^{\text {th }}$ April 2020.

17. Camara B, Kantambadouno JB, Martin-Blondel G, Berry A, Alvarez M, Benoit-Vical F, et al. Hyperreactive malarial splenomegaly: Three clinical cases and literature review. Med Mal Infect. 2009;39:29-35.

18. HenehanM, Montuno M, De Benedetto A. Doxycycline as an anti-inflammatory agent: updates in dermatology. 2017. J Eur Acad Dermatol Venereol. 2017;31(11):1800-1808.

19. Wang N, Tian X, Chen Y, Tan H, Xie P, Chen S, et al. Low dose doxycycline decreases systemic inflammation and improves glycemic control, lipid profiles, and islet morphology and function in $d b / d b$ mice. Sci Rep 7. 2017; 14707: https://doi.org/10.1038/s41598-017-14408-7

20. Betticher DC, Nicole A, Pugin P, Regamey C. The hyperreactive malarial splenomegaly syndrome in a European: has the treatment a modulatory effect on the immune system? J Infect Dis. 1990;161:157-159. 